\title{
Primary synovial sarcoma of the thyroid with locally repeated relapses in short periods: A case report
}

\author{
RONG-LIANG SHI ${ }^{1-3^{*}}$, NING QU ${ }^{*}$, LI-LI GAO ${ }^{2,4^{*}}$, ZHONG-WU LU $^{1}$, GUO-HUA SUN ${ }^{1}$ and QING-HAI JI ${ }^{1}$ \\ ${ }^{1}$ Department of Head and Neck Surgery, Fudan University Shanghai Cancer Center; ${ }^{2}$ Department of Oncology, \\ Shanghai Medical College, Fudan University, Shanghai 200032; ${ }^{3}$ Department of General Surgery, Minhang Hospital, \\ Fudan University, Shanghai 201199; ${ }^{4}$ Department of Pathology, Fudan University \\ Shanghai Cancer Center, Shanghai 200032, P.R. China
}

Received January 18, 2016; Accepted April 19, 2016

DOI: $10.3892 /$ br. 2016.670

\begin{abstract}
The primary occurrence of synovial sarcoma (SS) in the thyroid is quite rare. As other SS arise from the head and neck structure, it tends to present poor biological behaviors and is generally treated as a high-grade sarcoma. The present study reports the case of a 31-year-old male who presented a neck mass, involving the thyroid, as shown by ultrasonography. The tumor was resected by total thyroidectomy and diagnosed as SS by histopathology. However, the initial surgery was considered as incomplete (R2) and no adjuvant protocol was followed. At the follow-up, neck recurrences within local lymph nodes were found repeatedly. The tumor grade increased for the metastatic lesions, indicating poorer differentiations with repeated relapses. The accurate evaluations of the primary tumor facilitated it to tailor the initial treatments, otherwise, the prognosis may be deteriorated by inappropriate management.
\end{abstract}

\section{Introduction}

Synovial sarcoma (SS) is a type of mesenchymal tissue cell tumor exhibiting aggressive features, such as local invasiveness and distant metastasis (1). SS occurs 10-20\% in the soft tissue of the extremities with an onset in the pediatric and adult age ranges $(2,3)$. The head and neck region is the second most frequent site, representing $\sim 5 \%$ of the cases (4); thus, primary thyroid SS occurrence is quite rare. As is known, the

Correspondence to: Dr Qing-Hai Ji, Department of Head and Neck Surgery, Fudan University Shanghai Cancer Center, 270 Dong An Road, Shanghai 200032, P.R. China

E-mail: jiqinghai@shca.org.cn

${ }^{*}$ Contributed equally

Abbreviations: SS, synovial sarcoma; LN, lymph node; IHC, immunohistochemistry

Key words: synovial sarcoma, thyroid gland, neoplasm, treatment, prognosis conventional risk factors for the poor prognosis of SS include the clinicopathological features, such as tumor size and site; however, a previous study reported that the prognosis for SS patients depends largely on the feasibility and radicalness of surgical resection (5). The appropriate initial managements provide a good chance of a cure for localized disease; otherwise, it may lead to early or late recurrences (6). The present study reports a case of primary thyroid SS with repeated local relapses, examined the experience and combined this with a review of the literature, with the aim to improve the understanding of the appropriate initial management for this rare type of tumor.

\section{Case report}

Clinical findings and diagnostic focus. A 31-year-old male patient presented with a large mass in the right-anterior neck for 1 month without any symptoms. There was no noteworthy past and family history. The patient was referred to a local hospital based on the cervical findings of the neck mass by palpitation and ultrasonography. Physical examination revealed a $5 \mathrm{~cm}$ in diameter, firm, dipping thyroid mass. No cervical lymphadenopathy was observed. Ultrasonography revealed a large mass with an area of $5 \times 2 \times 2 \mathrm{~cm}^{3}$ in the right thyroid lobe, which was heterogeneous, hypervascular and solid. The biological thyroid function tests and serum tumor markers were normal. Written informed consent was obtained from the family of the patient for this case report and any accompanying images.

Therapeutic focus, histological assessment and follow-up. Initial treatment and assessment. Total thyroidectomy was performed as the initial procedure due to the suspicion for poorly differentiated tumor, without preoperative cytology. Thyroidectomy was performed without lymph node (LN) dissection. Intraoperative exploration revealed that the left thyroid lobe was hypertrophic and firm; the right lobe was under tension and enlarged, infiltrating irregularly into the surrounding soft tissues. The mobilization of the mass was difficult, leading to its capsular rupture and effusion of hemorrhagic from lobe and muscles. Due to this, the resection was classified as incomplete (R2). A postoperative histological examination showed that the tumor contained two 

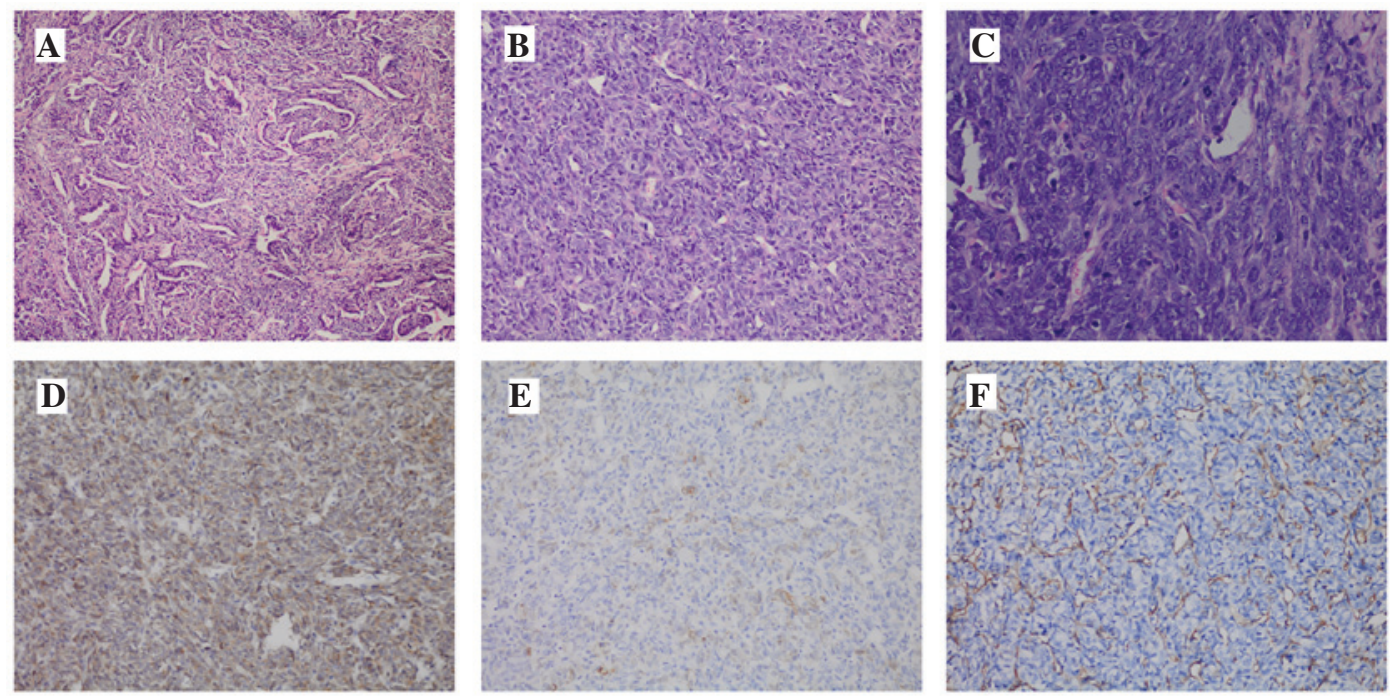

Figure 1. Pathology and immunohistological staining of the removed tumor and metastatic lesions. (A) The tumor at first surgery appeared biphasic, containing spindle cells and numerous glandular structures lined by well-differentiated cuboidal epithelium (H\&E; magnification, x100). (B and C) The recurred tumor in the right neck at the second and third surgeries had a poorly differentiated appearance, characterized by predominantly undifferentiated round cell morphology [(B) H\&E, magnification, x100; (C) H\&E, magnification, x200]. (D) The tumor cells were diffusely positive for B-cell lymphoma-2. (E) The glandular structures showed weak positivity for cytokeratin. (F) The glandular structures were positive for vimentin. H\&E, hematoxylin and eosin.
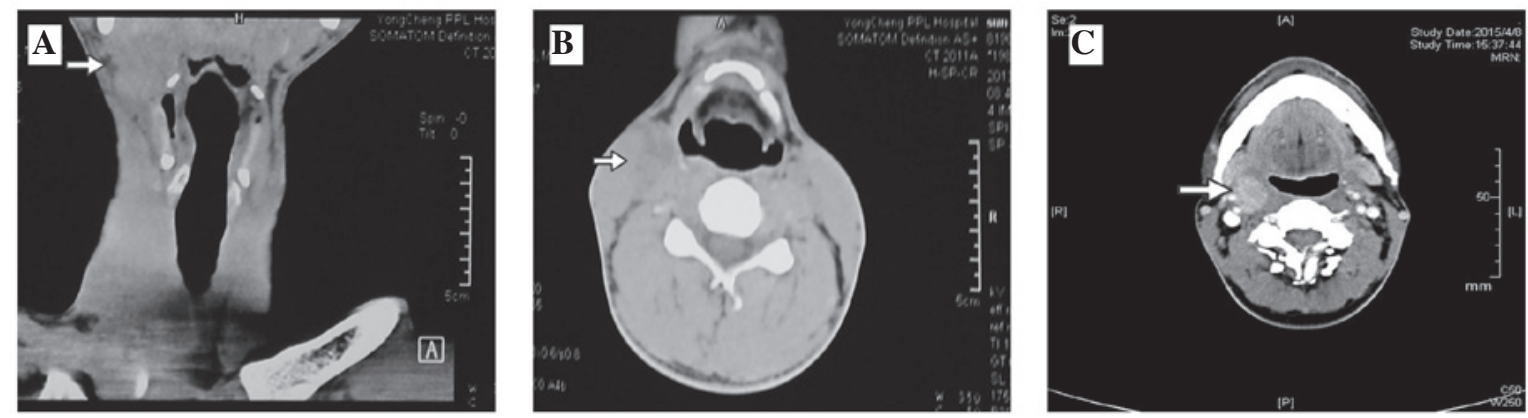

Figure 2. Images of the neck recurrences. (A and B) A follow-up CT scan in the 2 years after initial treatments showing a $2.2 \times 2-\mathrm{cm}^{2}$ metastatic lymph node with relatively clear borders in the right upper neck area. (C) Contrast-enhanced CT scan in the 10 months after the first recurrence, showing a repeated lymphadenopathy with contrasted disproportionation remaining in the right upper neck, which was suspected to relapse for the second time. CT, computed tomography.

components; spindle cells and glandular structures (Fig. 1A). The immunohistochemistry (IHC) examination showed that the tumor cells were positive for B-cell lymphoma-2 and cytokeratin (Fig. 1D-F). Finally, the diagnosis was biphasic SS. The resection margins (right sternocleidomastoids) were microscopically involved by tumor cells. No further surgery or treatment was chosen for the patient at the local hospital.

First neck recurrence and histology transformation. Two years after surgery, a follow-up computed tomography (CT) scan at a local hospital showed a patchy $\mathrm{LN}$ measuring $\sim 2 \mathrm{~cm}$ in diameter in the right upper neck, while the neck structure was clear (Fig. 2A and B). The histological examination from the excision biopsy revealed the growth of spindle-shaped, ovoid or polygonal neoplastic cells with irregular nuclei. The glandular structures were absent, which were compatible with a histological diagnosis of poorly differentiated SS (Fig. 1B). Adjuvant chemotherapy containing ifosfamide $\left[2,500 \mathrm{mg} / \mathrm{m}^{2}\right.$, continuous intravenous administration (CIV), days 1-3] and doxorubicin $\left(20 \mathrm{mg} / \mathrm{m}^{2}, \mathrm{CIV}\right.$, days 1-3) was administered every 3 weeks for four cycles. A total of $50 \mathrm{~Gy}$ of adjuvant radiotherapy was applied to the bed of the metastatic LN with the hope of an increased recurrence-free survival. The patient completed treatment and continued to undergo follow-up.

Second neck recurrence with poorly differentiated histology. A neck contrast-enhanced CT scan was performed 10 months after the first recurrence. It showed a voluminous LN in the right upper neck with contrasted disproportionation and unclear margins, $\sim 2.5 \mathrm{~cm}$ in diameter (Fig. $2 \mathrm{C}$ ). Thus, the patient was referred to the Fudan University Shanghai Cancer Center (Shanghai, China). A therapeutic level II regional LNs dissection was performed based on the history of disease and imaging findings, and we found that the spindle tumor cells appeared more crowded, with more apparent pathological mitotic figures (Fig. 1C). The pathological findings indicated that the tumor grade increased compared to the previous ones.

\section{Discussion}

SS is generally considered a high-grade, aggressive sarcoma. It is noted for its propensity for late local recurrence and 
metastasis, with the latter often occurring $>5$ years. Here we reported an adult case of small primary thyroid SS $(\leq 5 \mathrm{~cm})$, but with poor biological behavior and differentiated progression following the initial treatment. Based on the publication of an Italian series in 2009, tumor site was included as one of the variables for risk stratification purposes, and tumors arising from the head-neck were classed as 'high-risk' regardless of any other clinical features (7). Multidisciplinary treatments should depend on the risk stratification; otherwise, inappropriate treatment may deteriorate the prognosis, particularly in 'high-risk' patients. From the present case, data on the prognosis that showed repeated recurrences in short periods were collected. In combination with reviewing the previous studies, experience was gained regarding the accurate diagnosis and optimal treatment of primary thyroid SS.

First, it is important to combine imaging and pathology exams to assist in locating the tumor and establishing a diagnosis. In the present case, the initial symptom was the accidental identification of a thyroid mass without common symptoms. The asymptomatic clinical manifestation is easily ignored and misdiagnosed. In the present situation, imaging exams and fine-needle aspiration cytology may be useful for the diagnosis and staging of tumors. However, the patient was not diagnosed preoperatively at the initial surgery. Furthermore, no information on the initial staging was provided. When a malignant thyroid disease was suspected, a certain type of staging (ultrasound of the neck, magnetic resonance imaging or CT) could have been performed for the purpose of localization on local relapse structuring with the large vessels and tracheo-asophageal ducts. In the present case, CT scans provided evidence and guided the re-surgeries when tumor relapsed on various occasions.

Second, the recognition and differential diagnosis are difficult when SS arises in an uncommon site such as the thyroid. Generally, the diagnosis is based on pathological, IHC and molecular analyses of the resected specimen. Such cases could be discussed in a multidisciplinary oncological board preferably prior to surgery and particularly following surgical resection with positive margins. In the present case, histological examination well differentiated the SS according to typical features; additionally, it suggested that the tumor grade increased for the metastatic neck lesions, which was a reflection of poorer differentiations with repeated relapses and the importance of initial treatment to prognosis in SS. Although morphological and IHC examinations could be unequivocal for the diagnosis of SS, the molecular analysis detecting the SYT-SSX gene fusion, that is reported to be positive $>95 \%$ of $\mathrm{SS}$, is an alternative approach (8). Such analysis is particularly feasible in the case of diagnostic uncertainty on the preoperative diagnosis of SS.

Third, although primary thyroid SS has limited clinical data and no standardized treatments according to current studies, complete surgical excision remains the cornerstone of treatments. Due to the indetermination of the neck mass, the large tumor size and invasion of neighbor tissues, wide margin-free surgical resection (R0) was not achieved in this case. Subsequently, surgical revision could have been offered following positive margins. Negative margins and adjuvant radiotherapy, particularly in those patients with positive histological margins, are known to govern the local recurrence-free survival in neck SS (9). Intensive follow-up, instead of adjuvant radiotherapy, was delivered following the initial surgery in this case. Although SS is recognized for its relative chemosensitivity, the chemotherapy is an additional tool used for subclinical lesions and is particularly important for prevention from distant metastasis. In the present case, chemotherapy was involved in the therapeutic protocol when the tumor relapsed. Although the effect was not significant in the local disease control, no distant metastasis was observed in the follow-up period. In contrast with adjuvant therapy, the use of neoadjuvants is likely to reduce tumor size and offer surgical conditions for patients who cannot undergo surgery immediately, improving radical surgical resection rates. The adjuvant chemotherapy for this adjunct would have been better following the initial surgery. Therefore, surgery, radiotherapy and chemotherapy may be administered separately or in combination in the treatment for neck SS. The lessons from the present case indicated that multidisciplinary treatment should be tailored to the exact initial evaluation diagnosis, so as to improve the prognosis referring to local or distant control following initial management.

Finally, SS commonly metastasizes to the lungs and pleura, and other sites of metastasis include bone and LN. In the present case, neck LNs were involved as local recurrences within a short period twice. When lymphadenopathy was detected the first time, LN biopsy without regional resection was performed. Removing a LN in this case was not appropriate, as it was reported as an $\mathrm{R} 2$ situation due to the spillage of tumor material and positive resection margins on histopathology. Even if this intention was to confirm the presence of a recurrence, nodal dissection with resection of the rest thyroid or peri-thyroid tissues would have been the correct intervention unless the patient denied additional surgery. Although radio- and chemotherapy were followed on the confirmation of node metastasis, a repeated recurrence was again identified within the neck nodes. This emphasizes the role of complete surgical excision not only for the primary lesions, but also for the locally resectable recurrences.

In conclusion, thyroid SS is a malignant tumor with a low incidence, no specific clinical manifestations and lack of unified and effective treatments. Accurate assessment of the disease preoperatively allows for possible tailoring of the treatment. Radical surgical resection is fundamental and benefits disease control, otherwise, it may deteriorate the prognosis and differentiated progression. The multimodality protocol potentially improves local and distant tumor controls, but requires further investigation with reliable data.

\section{Acknowledgements}

The present study was supported by funds from the National Science Foundation of China (grant nos. 81572622 and 81272934 to Dr Qing-hai Ji) and the Natural Science Foundation of Shanghai (grant no. 12JC1402802 to Dr Qing-hai Ji).

\section{References}

1. Fisher C: Synovial sarcoma. Ann Diagn Pathol 2: 401-421, 1998. 2. Pappo AS, Fontanesi J, Luo X, Rao BN, Parham DM, Hurwitz C, Avery L and Pratt CB: Synovial sarcoma in children and adolescents: The St Jude Children's Research Hospital experience. J Clin Oncol 12: 2360-2366, 1994. 
3. Ferrari A, De Salvo GL, Brennan B, van Noesel MM, De Paoli A Casanova M, Francotte N, Kelsey A, Alaggio R, Oberlin O, et al: Synovial sarcoma in children and adolescents: the European Pediatric Soft Tissue Sarcoma Study Group prospective trial (EpSSG NRSTS 2005). Ann Oncol 26: 567-572, 2015.

4. Al-Daraji W, Lasota J, Foss R and Miettinen M: Synovial sarcoma involving the head: Analysis of 36 cases with predilection to the parotid and temporal regions. Am J Surg Pathol 33: 1494-1503, 2009.

5. Okcu MF, Munsell M, Treuner J, Mattke A, Pappo A, Cain A, Ferrari A, Casanova M, Ozkan A and Raney B: Synovial sarcoma of childhood and adolescence: A multicenter, multivariate analysis of outcome. J Clin Oncol 21: 1602-1611, 2003.

6. Sultan I, Rodriguez-Galindo C, Saab R, Yasir S, Casanova M and Ferrari A: Comparing children and adults with synovial sarcoma in the surveillance, epidemiology, and end results program, 1983 to 2005: An analysis of 1268 patients. Cancer 115: 3537-3547, 2009.
7. Orbach D, Mc Dowell H, Rey A, Bouvet N, Kelsey A and Stevens MC: Sparing strategy does not compromise prognosis in pediatric localized synovial sarcoma: Experience of the International Society of Pediatric Oncology, Malignant Mesenchymal Tumors (SIOP-MMT) Working Group. Pediatr Blood Cancer 57: 1130-1136, 2011.

8. Thway K and Fisher C: Synovial sarcoma: Defining features and diagnostic evolution. Ann Diagn Pathol 18: 369-380, 2014.

9. Harb WJ, Luna MA, Patel SR, Ballo MT, Roberts DB and Sturgis EM: Survival in patients with synovial sarcoma of the head and neck: Association with tumor location, size, and extension. Head Neck 29: 731-740, 2007. 